\title{
Re-audit of variations in written consent for Laparoscopic Cholecystectomy and Inguinal Hernia Repair
}

Poster presented at BAPIO National Conference, London 2019

Peer reviewed by Subarna Chakravorty PhD \& Sunil Daga PhD BAPIOAC19-P08

Evidence for the existence of the FGFR1-NMDAR2B heteroreceptor complexes in the rat brain, and the relevance of this in treating major depressive disorder

Ashwin Venkatesh, University of Cambridge, UK

Cite as: Venkatesh,A. Evidence for the existence of the FGFR1-NMDAR2B heteroreceptor complexes in the rat brain, and the relevance of this in treating major depressive disorder. The Physician 2020 vol4;issue1:abp08
Vikramman Vignaraja

Cite as: Vignaraja, V. (2020) Re-audit of variations in written consent for Laparoscopic Cholecystectomy and Inguinal Hernia Repair. The Physician vol 6; issue 2; epub 01 Jan 2020; DOI: https://doi.org/10.38192/1.6.2.12

Article Information

Submitted $\quad 01$ Nov 2019

Published 01 Aug 2020

Open Access - Creative Commons Licence CC-BYND-4.0

\section{Background:}

Major depressive disorder (MDD) is both a ubiquitous yet heterogeneous condition, affecting around $15 \%$ of individuals at least once in their lives, who may present with an array of physical and cognitive symptoms. Accumulating evidence posits that the N-methyl-Daspartate (NMDA) receptor may play an important underlying role in the neurobiology and thus the treatment of this disease. In addition, the FGFR1 receptor tyrosine kinase was previously shown to produce antidepressant-like effects when bound by one of its ligands. The formation of FGFR1-NMDA heteroreceptor complexes through allosteric receptorreceptor interactions may modulate downstream signalling pathways as well as recognition, trafficking and internalization of the constituent receptor protomers. Therefore we proposed the existence of a state of equilibrium between the FGFR1 and NMDA receptors and their heteroreceptor complex in the neuronal membrane, the balance of which may be altered in the depressed state.

\section{Methods:}

In our experiments, we used hippocampal and raphe nuclei sections from the brains of a wild-type SpragueDawley (SD) rat and in a selectively bred genetic rat model of depression, the Flinders Sensitive Line (FSL) rat, for comparison. We then localized and quantified the receptor complexes through immunofluorescence and in situ proximity ligation assay (PLA) techniques.

\section{Results and Conclusions:}

We obtained evidence for the existence of FGFR1-NMDAR2B heteroreceptor complexes in the hippocampus and midbrain areas of the adult rat brain. Analysis of the FGFR1 and NMDAR2B immunoreactivities indicate strong co-distribution of both receptor protomer in the same brain areas. Interestingly, NMDAR2B immunoreactivity studies suggest also a reduction in the expression level of the NMDAR2B subunit in the hippocampus and raphe brain regions of the FSL adult rats. Preliminary quantification analysis showed a significant reduction in the positive FGFR1NMDAR2B PLA clusters in both studied areas in the genetic animal model for depression (FSL) with the 
exception of the molecular cell layer of the dentate gyrus. Taken together, this concept could pave new avenues for therapeutic exploration in reducing the global occurrence, recurrence and severity of major depressive disorder. 